\title{
Clinical implications of aberrant neurovascular structures coursing through the submandibular gland
}

\author{
Kelsey J Eaton ${ }^{1}$, Heather F Smith ${ }^{\text {Corresp. } 2,3}$ \\ ${ }^{1}$ Department of Osteopathic Manipulative Medicine, Midwestern University, Glendale, Arizona, United States \\ 2 Department of Anatomy, Midwestern University, Glendale, Arizona, United States \\ ${ }^{3}$ School of Human Evolution and Social Change, Arizona State University, Tempe, Arizona, United States \\ Corresponding Author: Heather F Smith \\ Email address: hsmith@midwestern.edu
}

Background: Variation within the submandibular triangle, including variant paths of facial neurovasculature, could increase risk of neurovascular derangement during submandibular gland (SMG) dysfunction, enlargement, interventions, or removal. Methods: Frequency of anatomical variants enveloped within or piercing the SMG, including facial artery, vein, or branches of CN VII, were assessed in 70 cadaveric submandibular glands (39M/31F).

Results: Eighteen of 70 SMGs (25.7\%) were pierced by at least one aberrant neurovasculature structure: Facial artery most frequently $(n=13)$, followed by facial vein $(n=2)$, inferior labial artery and vein $(n=1)$, and CN VII cervical branch $(n=1)$. This study demonstrated the high variability of neurovasculature within submandibular parenchyma. These aberrant neurovascular structures, especially facial artery, are in danger of compromise during surgical and other medical procedures on the SMG. To avoid potential neurovascular compromise, ultrasonographic or other imaging is recommended prior to procedures involving the SMG. 
1 Clinical implications of aberrant neurovascular structures 2 coursing through the submandibular gland

3

4 Kelsey James Eaton ${ }^{1}$, Heather Frances Smith ${ }^{2,3 *}$

$5{ }^{1}$ Department of Osteopathic Manipulative Medicine, Midwestern University, Glendale, AZ,

6 USA

72 Department of Anatomy, Midwestern University, Glendale, AZ, USA

$8{ }^{3}$ School of Human Evolution and Social Change, Arizona State University, Tempe, AZ, USA

9

10 Corresponding Author:

11 Heather F. Smith, Ph.D. ${ }^{1}$

12 Department of Anatomy, Midwestern University, 19555 N. 59th Ave., Glendale, AZ 85308, 13 USA.

14 Email address: $\underline{\text { hsmith@midwestern.edu }}$

15 


\section{Abstract}

17

18 Background: Variation within the submandibular triangle, including variant paths of facial

19 neurovasculature, could increase risk of neurovascular derangement during submandibular gland

20 (SMG) dysfunction, enlargement, interventions, or removal.

21 Methods: Frequency of anatomical variants enveloped within or piercing the SMG, including

22 facial artery, vein, or branches of CN VII, were assessed in 70 cadaveric submandibular glands

$23(39 \mathrm{M} / 31 \mathrm{~F})$.

24 Results: Eighteen of 70 SMGs (25.7\%) were pierced by at least one aberrant neurovasculature

25 structure: Facial artery most frequently $(\mathrm{n}=13)$, followed by facial vein $(\mathrm{n}=2)$, inferior labial

26 artery and vein $(\mathrm{n}=1)$, and CN VII cervical branch $(\mathrm{n}=1)$. This study demonstrated the high

27 variability of neurovasculature within submandibular parenchyma. These aberrant neurovascular

28 structures, especially facial artery, are in danger of compromise during surgical and other

29 medical procedures on the SMG. To avoid potential neurovascular compromise,

30 ultrasonographic or other imaging is recommended prior to procedures involving the SMG. 


\section{Introduction}

34 The submandibular triangle contains a complex interaction of anatomy and physiology, allowing

35 for numerous health conditions and procedures to occur. This well-defined region is outlined by

36 the inferior border of the mandible and the anterior and posterior bellies of the digastric muscle

37 (Figure 1). The superficial and deep boundaries are limited by the platysma and the mylohyoid

38 muscle, respectively, creating a limited space for its many contents. The submandibular gland

39 (SMG), also known as the submaxillary gland, typically occupies the majority of the

40 submandibular triangle.

41 The SMG is vulnerable to eclectic pathology that involves both clinical and surgical

42 interventions $s^{1,2,3,4}$. Enlargement of the gland may occur, most commonly due to salivary flow

43 obstruction, infection, autoimmune disorders, and iatrogenic consequences. The current standard

44 of care for enlargement suspicious for neoplastic origin is Fine Needle Aspiration (FNA). This

45 procedure samples the gland contents; however, its findings may result in indefinite answers due

46 to the nature of the sampling technique as FNA has varying reported rates of sensitivity ${ }^{5}$. While

47 neoplastic occurrence is relatively rare in the SMG, positive diagnostic findings typically result

48 in removal of the gland. Thus, for patients with positive or inconclusive testing neoplastic, the

49 treatment plan begins with removal of the gland ${ }^{6,7,8}$. Patients with neurological deficits such as

50 cerebral palsy can similarly be affected by iatrogenic changes to the SMG. Those who suffer

51 from sialorrhea, an overproduction of saliva, may undergo Botox injection to minimize saliva

52 production, ligation of the submandibular salivary duct, or even complete SMG removal to help

53 alleviate refractory symptoms ${ }^{9}$. Finally, the SMG's shape or position can be altered for aesthetic

54 correction from SMG ptosis ${ }^{10}$. 
The pathologies and procedures of the submandibular triangle occur with varying

56 frequency; however, care providers must be aware of the restrictions and variances within this

57 space. Accurate knowledge of the contents and surroundings of the SMG will facilitate designing

58 appropriate surgical planning, and possible explanation, prevention, or treatment of sequelae

59 following structural derangement within the submandibular triangle.

60 Given the relatively small size of the submandibular anatomical space and complex

61 multi-staged developmental pattern, we hypothesize that there is higher likelihood of significant

62 variation of the neurovascular pathways associated with the SMG than previously reported.

63 Classically, vascular and neural considerations placed the facial artery within a posterior path

64 along an open ceiling canal of the SMG (Figure 1). However, aberrant pathways in which the

65 facial artery may deviate and course within the parenchyma of the gland could put the

66 vasculature and nerves at risk in cases of pathology, surgical procedures, or other derangement of

67 the submandibular gland. In fact, a few cases of the facial artery piercing the SMG have been

68 reported in the literature ${ }^{11,12}$. The integrity of the facial artery is also relevant given the

69 postsynaptic sympathetic periarterial plexus that travels on its external surface. These concerns

70 are in addition to the more well-known complications of the marginal mandibular branch of

71 facial nerve or other lower facial nerve branches that are at risk of iatrogenic damage that are due

72 to close proximity to the $\mathrm{SMG}^{13-14}$.

73

74 Materials \& Methods

75 Seventy cadaveric submandibular glands $(39 \mathrm{M} / 31 \mathrm{~F})$ from the gross anatomy teaching

76 laboratories at Midwestern University were assessed to determine the frequency of variance in

77 submandibular gland neurovasculature within the submandibular triangle (Figure 1). Following 
78 student dissection, the integrity and presence of the SMG was first evaluated, as the student 79 coursework required dissection through the gland or complete removal in some cases. Cadaver

80 inclusion depended on a minimum of unilateral presence of the gland. Additional dissection and

81 preparation were necessary to further reveal the SMG and its associated neurovascular structures

82 in several cadavers. Careful dissection was conducted to assess whether any neurovascular

83 structures were coursing through the gland tissue, as opposed to coursing posteriorly and

84 adhering to the deep surface of the gland.

85 Data were then collected on the presence and frequency of neurovasculature enveloped 86 within the SMG. Neurovascular structures excluded from consideration were minor branches

87 directly off the facial artery and the marginal mandibular nerve of CN VII, as these are

88 considered known and accepted variants. For each gland, data on sex and laterality of variants

89 were also recorded. Chi-squared statistical analyses were conducted in SPSS 19 (IBM Corp.) to

90 determine whether significant differences existed in frequency of variants between sexes or sides

91 of the body. This study was determined to be IRB-exempt by the Midwestern University

92 Institutional Review Board, due to the subjects being entirely cadaveric (Exemption \#AZ1121).

93

\section{Results}

95 In the seventy preserved SMGs, 18 submandibular glands $(25.7 \%)$ were found to demonstrate 96 variations from the classical anatomy (Table 1). One of these glands contained two variations

97 within it, allowing for a total of 19 variant structures (Table 1). Variations were defined as

98 neurovasculature not classically associated with direct gland innervation or blood supply. Most

99 variants were found to be present unilaterally, with only one female cadaver possessing bilateral 100 variation. 
The predominant variation observed was the facial artery encapsulated by the SMG, 102 rather than traveling posteriorly, which comprised $68.4 \%$ of the observed variations $(n=13)$

103 totaling $18.6 \%$ of all SMGs (Table 2; Figure 2). This variant was followed in frequency by the

104 facial vein $(\mathrm{n}=2)$, inferior labial artery and vein $(\mathrm{n}=1)$, and cervical branch of CN VII $(\mathrm{n}=1)$

105 piercing the gland (Table 2). There were two additional unidentified neurovascular bundles

106 observed within gland parenchyma that had been severed from previous dissection. These

107 unidentified bundles were traced proximally and confirmed as being neither the glandular

108 branches from the facial artery nor the marginal mandibular nerve, which were both intact.

109 Chi-squared tests revealed significant sex differences in the frequency of neurovascular

110 anomalies. Female subjects displayed a significantly higher overall rate of piercing structures

111 compared to males $\left(12 / 31=38.7 \%\right.$ and $6 / 39=15.4 \%$, respectively), $\chi^{2}=4.92, p=0.027$.

112 Females also demonstrated a significantly higher rate of the facial artery piercing the SMG (9/33

$113=27.3 \%)$ than males $(4 / 39=10.3 \%)$ with $\chi^{2}=4.03, p=0.045$. Comparisons of the frequency of

114 variants between the left and right sides were insignificant for overall variants $\left(\chi^{2}=0.14, p=\right.$

$1150.71)$, and for the facial artery $\left(\chi^{2}=0.34, p=0.56\right)$. The low frequencies in the other

116 neurovascular variants yielded sample sizes that were insufficient for statistical comparisons.

\section{Discussion}

118 The previously accepted anatomical pattern of the submandibular triangle presumed that the

119 facial artery courses via a posterior canal alongside the SMG as it provided blood supply via

120 glandular branches ${ }^{15}$. A prevalence of variation in this arterial path has not been widely

121 established. The high frequency of aberrant anatomy revealed in this study $(25.7 \%)$ indicates that

122 there is greater variation in the submandibular triangle than commonly acknowledged. 
123 Additionally, we report for the first time that such variants are significantly more common in

124 females than in males.

125 Surgical procedures are the most obvious clinical application of these variant patterns;

126 however, many pathologies causing glandular enlargement are of both surgical and clinical

127 interest. These pathologies range from disorders such as neoplasms, diabetes, hypothyroidism,

128 autoimmune disorders, and viral infections $2,3,4,7$. When SMG enlargement occurs in patients with

129 variant neurovasculature compression by the gland may lead to theoretical nerve entrapment,

130 salivary consistency and production changes, and/or intraoperative complications dependent on

131 the structure enveloped ${ }^{16}$. Therefore, structural knowledge is essential in understanding each

132 patient's true functionality and pathology, especially those presenting with atypical symptoms or

133 those refractory to treatment. Clinically, the neurological structure previously considered most at

134 risk during common procedures involving the SMG was the marginal mandibular nerve $(\mathrm{CN}$

$135 \mathrm{VII})$ as it lies adjacent to the SMG with utilization of the facial vein and artery as landmarks for

136 preservation ${ }^{13,14}$. While the risk to the marginal mandibular nerve during surgery is well

137 documented, we believe that the above results provide sufficient evidence of other neurovascular

138 structures at risk that must be considered prior to interventions in this region.

139 Anatomically, knowledge of neural and vascular location is pivotal within surgical

140 specialties. The facial artery may be readily sacrificed in operations if necessary, for

141 advancement, visualization, or mobilization ${ }^{17}$. Due to the amount of collateral blood flow,

142 removal of the facial artery has no clinical consequence outside of hematoma risk from ligation

143 failure or infections ${ }^{18}$. However, knowledge of this piercing variant is important after trauma or

144 locally invasive neoplasm as the facial artery may be used for facial flaps in follow-up

145 reconstructive procedures ${ }^{19}$. 
147 and tissues become increasingly lax with age, the submandibular gland can experience ptosis,

148 creating undesired enlargement under the mandible ${ }^{10,18}$. Several procedures are of note,

149 including SMG suspensions and shaving. The former cosmetic procedure elevates the glands

150 from below the mandible providing a rejuvenating appearance and does not routinely involve

151 ligation of the facial artery since the SMG is preserved ${ }^{10}$. A shaving technique with similar

152 results involves surgical contouring of the SMG, which includes explicit concerns over the

153 location of the facial artery and its penetrating branches ${ }^{18}$.

154 Clinically, we postulate several reasonable consequences or potential complications due

155 to these newly identified anatomical variants in the submandibular triangle. Case reports have

156 shown pseudoaneurysm occurrence with botulinum (Botox) injection or from trauma to vessels

157 of the neck and face 20,21 . The procedure is associated with varying commitment to

158 ultrasonography of the gland's position and structure prior to injection which may lead to

159 inaccurate placement of the needle ${ }^{22}$. Knowledge of these anatomical variants and the possibility

160 of pseudoaneurysm and undesired vasodilatory effects predicates the necessity of consistently

161 using ultrasound prior to injection so as to avoid neural or vascular structures. This may be

162 especially true with many of the populations that require SMG interventions as they may have

163 already poor nutritional status or previous radiotherapies, which could predicate pseudoaneurysm

164 formation $^{23}$.

165 Salivary tumors probability of malignancy changes in relation to the gland. The accepted

166 rule of thumb is that with decreasing size of the gland, there is an increased incidence of

167 malignancy ${ }^{6}$. This is demonstrated by the $25 / 50 / 75$ rule for parotid, submandibular, and

168 sublingual glands respectively ${ }^{6}$. Dependent on tumor subtype salivary gland neoplasms may 
169 spread preferentially either using lymphangiogenesis or hematogenously with distant metastasis

$170{ }^{24}$. A neoplasm arising in an SMG that contains a facial artery, vein, or other aberrant structure

171 could have additional consequences and considerations such as metastasis risk or compression.

172 Arterial walls are more resistant to infiltration or compression by neoplastic cells in comparison

173 to the thinner-walled veins. However, as mentioned previously, the facial artery transports a

174 periarterial postganglionic sympathetic nerve plexus on its external walls, which would be at

175 risk. Certain neoplasms such as adenoid cystic carcinomas, the most common location being in

176 the minor and major salivary glands, can spread along these nerves in a perineural manner well

177 before it is visible on imaging ${ }^{25,26}$.

178 Postganglionic sympathetic nerves may be at risk in non-malignant conditions as well.

179 Stricture, enlargement, or fibrosis such as in chronic sclerosing sialadenitis (Kuttner's Tumor)

180 could reasonably apply pressure to the sympathetic plexus over the facial artery. This

181 relationship may play a role in salivary dysfunction and warrants further study as the glands

182 receive input from both the sympathetic and parasympathetic systems ${ }^{27,28}$. Unlike much of the

183 rest of the body in which these systems have antagonistic effects, in the salivary glands, both

184 sympathetics and parasympathetics stimulate production of saliva. The difference between the

185 input of the two systems results in volume and consistency changes ${ }^{29}$.

186 For all procedures and interventions outlined above, an understanding of the

187 neurovascular anomalies reported here would allow for appropriate planning and risk-benefit

188 consideration. Being aware of the involvement of nerves and arteries coursing through the gland

189 can facilitate the improvement of informed consents when discussing the procedure and its

190 potential complications. In order to better understand the prevalence of aberrant anatomy within 
191 the submandibular region, further study could focus on a sample of live subjects using

192 ultrasonography to assess rates of anatomical variance.

193

194 Conclusions

195 This study revealed a high degree of variability in the neurovasculature within the submandibular

196 triangle, identifying the high frequency of anomalous facial artery patterns. As a result,

197 compression of these structures should be considered when treating a patient with lower head

198 and neck symptomatology. The data also indicate a statistically higher rate of aberrant

199 submandibular neurovasculature in females than in males. Knowledge of possible variance

200 allows for individualized and comprehensive treatment plans, more informed surgical approach,

201 and facilitation of more targeted procedures when necessary.

202

203 Acknowledgements

204 The authors would like to thank the generous body donors whose cadavers formed the basis of

205 this study. Figure 1 was created by Brent Adrian. Thank you to Ashley Bergeron and the

206 Anatomical Laboratories staff for their accommodation in the anatomy laboratories. We thank

207 Drs. Robert Druzinsky, David Reed, and an anonymous reviewer for constructive feedback that

208 improved the paper. The authors declare no conflict of interest.

209

210 References

211 1. Wilson KF, Meier JD, Ward PD. Salivary gland disorders. Am Fam Physician.

$212 \quad$ 2014;89(11):882-888. 
213 2. McQuone SJ. Acute viral and bacterial infections of the salivary glands. Otolaryngologic $214 \quad$ Clinics of North America. 1999;32(5):793-811.

215 3. Iro H, Zenk J. Salivary gland diseases in children. GMS current topics in 216 otorhinolaryngology, head and neck surgery. 2014;13.

217 4. Schiødt M, Dodd CL, Greenspan D, Daniels TE, Chernoff D, Hollander H, Wara D, 218 Greenspan JS. Natural history of HIV-associated salivary gland disease. Oral surgery, oral 219 medicine, oral pathology. 1992;74(3):326-331.

220 5. To VSH, Chan JYW, Tsang RK, Wei WI. Review of salivary gland neoplasms. ISRN $221 \quad$ otolaryngology. 2012;2012.

222 6. Agni NA, Borle RM. Salivary Gland Pathologies. JP Medical Ltd; 2013.

223 7. Smith W, Peters W, Markus A. Submandibular gland surgery: an audit of clinical findings, 224 pathology and postoperative morbidity. Annals of the Royal College of Surgeons of $225 \quad$ England. 1993;75(3):164.

226 8. Rapidis AD, Stavrianos S, Lagogiannis G, Faratzis G. Tumors of the submandibular gland: 227 clinicopathologic analysis of 23 patients. Journal of oral and maxillofacial surgery. $228 \quad 2004 ; 62(10): 1203-1208$.

229 9. Manrique D, Sato J. Salivary gland surgery for control of chronic pulmonary aspiration in 230 children with cerebral palsy. International journal of pediatric otorhinolaryngology. $231 \quad 2009 ; 73(9): 1192-1194$.

232 10. Sullivan PK, Freeman MB, Schmidt S. Contouring the aging neck with submandibular 233 gland suspension. Aesthetic surgery journal. 2006;26(4):465-471.

234 11. Vadgaonkar R, Rai R, Prabhu LV, Bv M, Samapriya N. Variant facial artery in the 235 submandibular region. Journal of craniofacial surgery. 2012; 23.4:e355-e357. 
236 12. Venugopal SV, Rao V, Kumar RB, Bhasin G. Relationship between the facial artery and 237 submandibular salivary gland. International journal of anatomical research. 2014; 2.3:597$238 \quad 600$.

239 13. Ichimura K, Nibu Ki, Tanaka T. Nerve paralysis after surgery in the submandibular triangle: 240 review of University of Tokyo Hospital experience. Head \& neck. 1997;19(1):48-53.

241 14. Bates D, O'brien CJ, Tikaram K, Painter DM. Parotid and submandibular sialadenitis treated 242 by salivary gland excision. Australian and New Zealand journal of surgery. 1998;68(2):120$243 \quad 124$.

244 15. Sidell D, Shapiro NL. Salivary Glands. In: Pediatric Surgery (Seventh Edition). Elsevier; $245 \quad 2012: 729-735$.

246 16. Ximenes Filho J, Imamura R, Sennes L. Neoplasias Benignas das Glândulas Salivares. Rev 247 Arq Int Otorrinolaringol. 2002;6(3):225-232.

248 17. Holsinger FC, Bui DT. Anatomy, function, and evaluation of the salivary glands. In: 249 Salivary gland disorders. Springer; 2007:1-16.

250 18. Mendelson BC, Tutino R. Submandibular gland reduction in aesthetic surgery of the neck: 251 review of 112 consecutive cases. Plastic and reconstructive surgery. 2015;136(3):463.

252 19. Talmi YP, Wolf M, Bedrin L, Horowitz Z, Dori S, Chaushu G, Yahalom R, Taicher S, 253 Kronenberg J. Preservation of the facial artery in excision of the submandibular salivary 254 gland. British journal of plastic surgery. 2003;56(2):156-157.

255 20. Dediol E, Manojlovic S, Biocic J, Franceski D, Ivanac G. Facial artery pseudoaneurysm 256 without evidence of trauma. International journal of oral and maxillofacial surgery. $257 \quad 2011 ; 40(9): 988-990$. 
258 21. Ribeiro-Ribeiro AL, Junior SdMA, Pinheiro JdJV. Traumatic pseudoaneurysm of the facial 259 artery: late complication and effects on local blood flow. Oral Surgery, Oral Medicine, Oral 260 Pathology, Oral Radiology, and Endodontology. 2011;112(1):e4-e9.

261 22. So JI, Song DH, Park JH, Choi E, Yoon JY, Yoo Y, Chung ME. Accuracy of ultrasound262 guided and non-ultrasound-guided botulinum toxin injection into cadaver salivary glands. 263 Annals of rehabilitation medicine. 2017;41(1):51-57.

264 23. Patel AK, Patterson JM, Chapple CR. Botulinum toxin injections for neurogenic and 265 idiopathic detrusor overactivity: a critical analysis of results. European urology. $2662006 ; 50(4): 684-710$.

267 268 269 270 271 272 273 274 275 276 277 278 279 280

24. Hertzer NR. Extracranial carotid aneurysms: a new look at an old problem. Journal of vascular surgery. 2000;31(4):823-825.

25. Fujita G, Sato S, Kishino M, Iwai S, Nakazawa M, Toyosawa S, Yura Y, Ogawa Y. Lymphatic vessels and related factors in adenoid cystic carcinoma of the salivary gland. Modern Pathology. 2011;24(7):885.

26. Vrielinck LJ, Ostyn F, Van Damme B, Van den Bogaert W, Fossion E. The significance of perineural spread in adenoid cystic carcinoma of the major and minor salivary glands. International journal of oral and maxillofacial surgery. 1988;17(3):190-193.

27. Jaso J, Malhotra R. Adenoid cystic carcinoma. Archives of pathology \& laboratory medicine. 2011;135(4):511-515.

28. Proctor GB, Carpenter GH. Regulation of salivary gland function by autonomic nerves. Autonomic Neuroscience. 2007;133(1):3-18.

29. Delfs U, Emmelin N. Degeneration secretion of saliva in the rat following sympathectomy. The Journal of physiology. 1974;239(3):623-630. 


\section{Figure 1}

Anatomy of the submandibular region illustrating the classic anatomical relationships of the submandibular gland and associated neurovasculature.

Abbreviations: $\mathrm{AD}=$ Anterior digastric; $\mathrm{CN} \mathrm{VII}=$ Facial nerve; $\mathrm{FA}=$ Facial artery; $\mathrm{FV}=$ Facial vein; PD = Posterior digastric; $\mathrm{PG}=$ Parotid gland; $\mathrm{SH}=$ Stylohyoid; $\mathrm{SMG}=$ Submandibular gland. Drawing source credit: Brent Adrian.

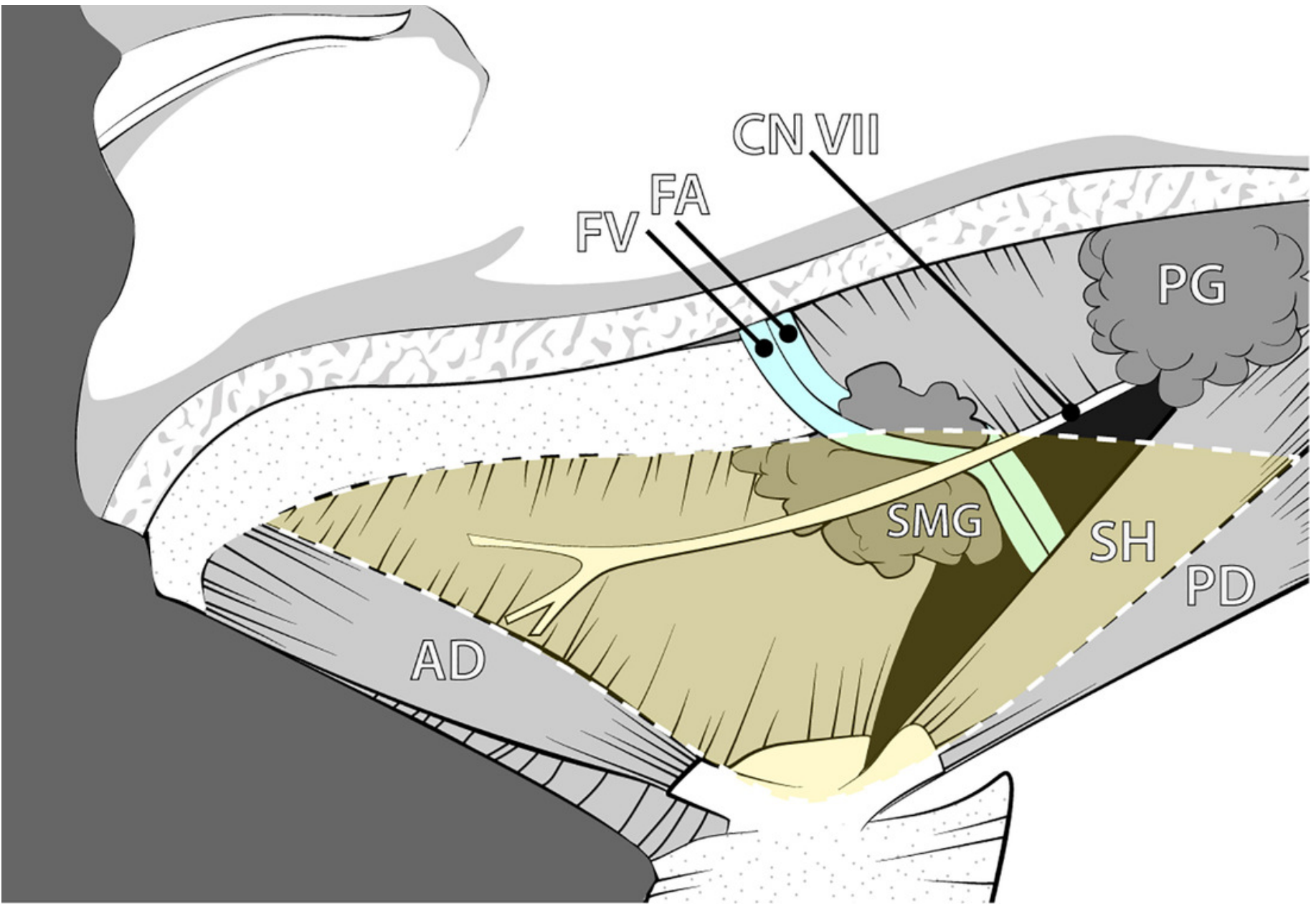




\section{Figure 2}

Photos demonstrating anatomical variations in neurovasculature around the submandibular gland

A) Anterior view of a hemisected cadaveric specimen demonstrating both classic (left side of photo) and variant (right side of photo) anatomy; B) Right SMG showing the classic anatomical condition in which no neurovasculature pierces the SMG; C) Left SMG showing a variant condition in which the facial artery courses through the SMG. Photo has been colored for easy identification of structures: Yellow = Submandibular gland; Red = Facial artery; Blue $=$ Facial vein .
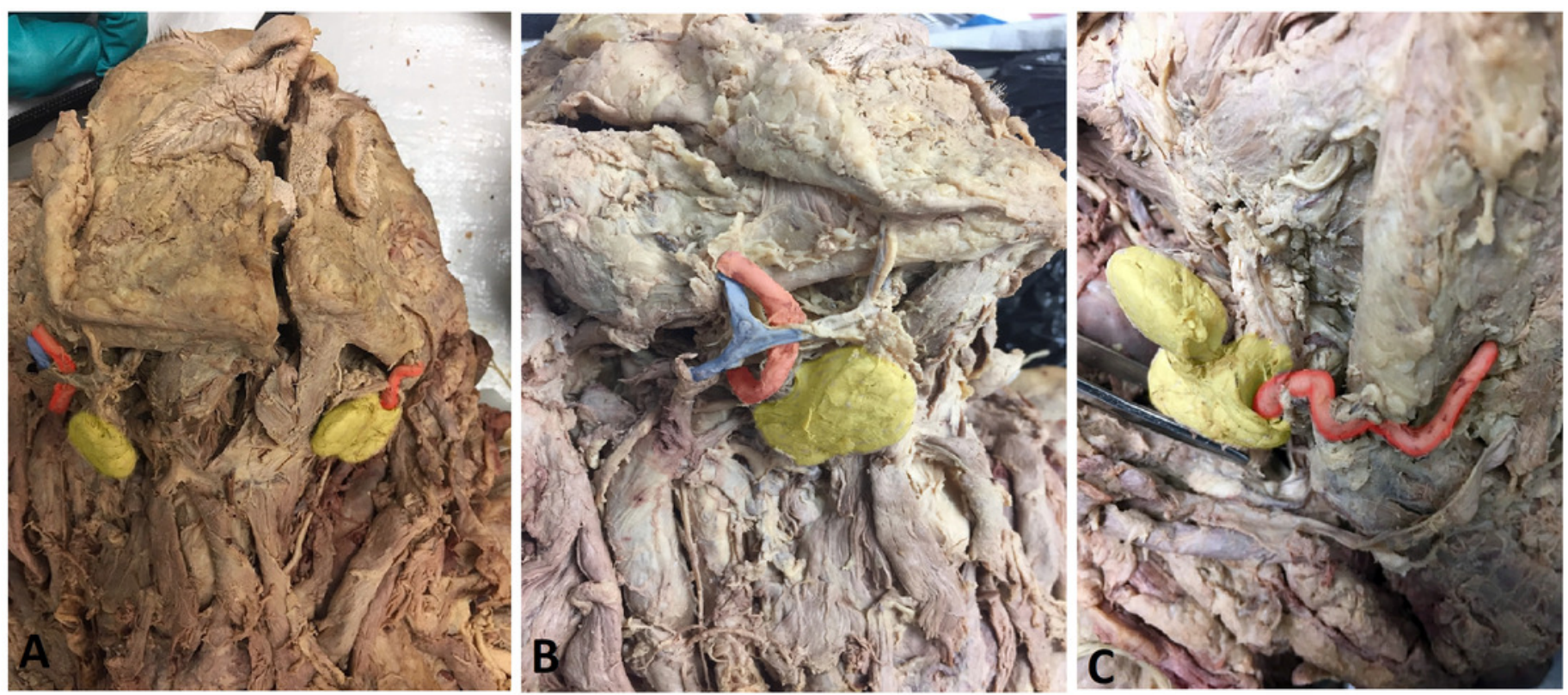


\section{Table $\mathbf{1}$ (on next page)}

Frequency of anatomically variant neurovascular structures within 18 of the 70 submandibular glands inspected.

Total variant structures were significantly more common in females than in males $\left(\chi^{2}=4.92\right.$, $p=0.027)$ * *nferior labial artery branched proximally, through the submandibular gland $\dagger$ One female cadaver had bilateral involvement of the facial artery in both SMGs. 


\begin{tabular}{|c|c|c|c|c|c|c|}
\hline Category & $\begin{array}{l}\text { Facial } \\
\text { artery }\end{array}$ & $\begin{array}{l}\text { Facial } \\
\text { vein }\end{array}$ & $\begin{array}{c}\text { Inferior } \\
\text { labial } \\
\text { artery* }\end{array}$ & $\begin{array}{l}\text { CN VII } \\
\text { cervical } \\
\text { branch }\end{array}$ & $\begin{array}{c}\text { Unidentified } \\
\text { neurovasculature }\end{array}$ & $\begin{array}{l}\text { Total variant } \\
\text { structures }\end{array}$ \\
\hline Male & 4 & 1 & 0 & 1 & 1 & 7 \\
\hline Female & $9+$ & 1 & 1 & 0 & 1 & 12 \\
\hline Left & 8 & 2 & 0 & 1 & 0 & 11 \\
\hline Right & 5 & 0 & 1 & 0 & 2 & 8 \\
\hline Total & 13 & 2 & 1 & 1 & 2 & 19 \\
\hline \multicolumn{7}{|c|}{$\begin{array}{l}\text { Table 1. Frequency of anatomically variant neurovascular structures within } 18 \text { of the } 70 \\
\text { submandibular glands inspected. Total variant structures were significantly more common in } \\
\text { females than in males }\left(X^{2}=4.92, p=0.027\right) \text {. } \\
\text { *Inferior labial artery branched proximally, through the submandibular gland } \\
\text { † One female cadaver had bilateral involvement of the facial artery in both SMGs. }\end{array}$} \\
\hline
\end{tabular}




\section{Table 2 (on next page)}

Sample size, laterality, and sex of cadaveric subjects with the most common anatomical variant of the submandibular gland, the facial artery piercing the SMG

The facial artery pierced the SMG in significantly higher frequency in females compared to

males $\left(\chi^{2}=4.03, p=0.045\right) . S M G=$ Submandibular Gland $*=$ One female cadaver displayed bilateral involvement of the SMGs 


\begin{tabular}{|c|c|c|}
\hline Category & Number of SMGs & Facial artery variants \\
\hline Male & 39 & $4(10.3 \%)$ \\
\hline Female & 31 & $9^{*}(29.0 \%)$ \\
\hline Left & 38 & $8(21.1 \%)$ \\
\hline Right & 32 & $5(15.6 \%)$ \\
\hline Total & 70 & $13(18.6 \%)$ \\
\hline \multicolumn{3}{|c|}{$\begin{array}{l}\text { Table 2. Sample size, laterality, and sex of cadaveric subjects with the most common } \\
\text { anatomical variant of the submandibular gland, the facial artery piercing the SMG. The facial } \\
\text { artery pierced the SMG in significantly higher frequency in females compared to males }\left(X^{2}=\right. \\
4.03, p=0.045) \text {. } \\
\text { SMG = Submandibular Gland } \\
\text { * = One female cadaver displayed bilateral involvement of the SMGs }\end{array}$} \\
\hline
\end{tabular}

2 